\title{
GRB 120422A: A LOW-LUMINOSITY GAMMA-RAY BURST DRIVEN BY A CENTRAL ENGINE
}

\author{
Bin-Bin Zhang ${ }^{1}$, Yi-Zhong Fan ${ }^{2,3}$, Rong-Feng Shen ${ }^{4}$, Dong Xu ${ }^{5,6}$, Fu-Wen Zhang $^{7}$, \\ Da-Ming WeI ${ }^{2,3}$, David N. Burrows ${ }^{1}$, Bing Zhang ${ }^{8}$, and NeIL Gehrels ${ }^{9}$ \\ ${ }^{1}$ Department of Astronomy and Astrophysics, Pennsylvania State University, University Park, PA 16802, USA; bbzhang@psu.edu \\ 2 Purple Mountain Observatory, Chinese Academy of Sciences, Nanjing 210008, China; yzfan@pmo.ac.cn \\ ${ }^{3}$ Key Laboratory of Dark Matter and Space Astronomy, Chinese Academy of Sciences, Nanjing 210008, China \\ ${ }^{4}$ Department of Astronomy \& Astrophysics, University of Toronto, M5S 3H4, Canada \\ ${ }^{5}$ Benoziyo Center for Astrophysics, Faculty of Physics, The Weizmann Institute of Science, Rehovot 76100, Israel \\ ${ }^{6}$ National Astronomical Observatories, Chinese Academy of Sciences, Beijing 100012, China \\ ${ }^{7}$ College of Science, Guilin University of Technology, Guilin, Guangxi 541004, China \\ ${ }^{8}$ Department of Physics and Astronomy, University of Nevada Las Vegas, Las Vegas, NV 89154, USA; zhang@physics.unlv.edu \\ ${ }^{9}$ NASA Goddard Space Flight Center, Greenbelt, MD 20771, USA \\ Received 2012 June 1; accepted 2012 July 17; published 2012 August 27
}

\begin{abstract}
GRB 120422A is a low-luminosity gamma-ray burst (GRB) associated with a bright supernova, which distinguishes itself by its relatively short $T_{90}(\sim 5 \mathrm{~s})$ and an energetic and steep-decaying X-ray tail. We analyze the $S w i f t$ Burst Alert Telescope and X-ray Telescope data and discuss the physical implications. We show that the steep decline early in the X-ray light curve can be interpreted as the curvature tail of a late emission episode around 58-86 s, with a curved instantaneous spectrum at the end of the emission episode. Together with the main activity in the first $\sim 20 \mathrm{~s}$ and the weak emission from $40 \mathrm{~s}$ to $60 \mathrm{~s}$, the prompt emission is variable, which points to a central engine origin in contrast to a shock-breakout origin, which is used to interpret some other nearby low-luminosity supernova GRBs. Both the curvature effect model and interpreting the early shallow decay as the coasting external forward shock emission in a wind medium provide a constraint on the bulk Lorentz factor $\Gamma$ to be around several. Comparing the properties of GRB 120422A and other supernova GRBs, we find that the main criterion to distinguish engine-driven GRBs from shock-breakout GRBs is the time-averaged $\gamma$-ray luminosity. Engine-driven GRBs likely have a luminosity above $\sim 10^{48} \mathrm{erg} \mathrm{s}^{-1}$.
\end{abstract}

Key words: gamma-ray burst: general - X-rays: general

Online-only material: color figures

\section{INTRODUCTION}

GRB 110422A triggered the Burst Alert Telescope (BAT; Barthelmy et al. 2005) on board Swift at 07:12:03 UT on 2012 April 22 (Troja et al. 2012). Swift slewed to the burst immediately. The two narrow-field instruments, the X-ray Telescope (XRT; Burrows et al. 2005) and the Ultraviolet Optical Telescope (UVOT; Roming et al. 2005), on board Swift began to observe the field at $T_{0}+95.1 \mathrm{~s}$ and $T_{0}+104 \mathrm{~s}$, respectively, where $T_{0}$ is the BAT trigger time. A bright X-ray afterglow was localized at R.A. $(\mathrm{J} 2000)=09^{\mathrm{h}} 07^{\mathrm{m}} 38^{\mathrm{s}} .46$, decl. $(\mathrm{J} 2000)=+14^{\circ} 01^{\prime} 05^{\prime \prime} 6$ with an uncertainty of $1^{\prime \prime} .9(90 \%$ confidence; Beardmore et al. 2012). A UVOT source was found within the XRT error circle (Kuin \& Troja 2012) and was confirmed by several ground follow-ups (e.g., Tanvir et al. 2012; Nardini et al. 2012; Rumyantsev et al. 2012). A redshift of $z=0.283$ was measured, and an associated supernova (SN) was soon discovered (Malesani et al. 2012a, 2012b; Melandri et al. 2012; Wiersema et al. 2012; Sanchez-Ramirez et al. 2012). This firmly places the burst in the massive star core-collapse category (Type II/long; Zhang et al. 2009a). An unusual property is the large offset of the gamma-ray burst's (GRB's) position from the center of its host galaxy, which is often interpreted as evidence of its compact-star-merger origin (Type I/short) (had the associated SN not been discovered). This might be related to massive star formation or death in an interacting system (Tanvir et al. 2012; Sanchez-Ramirez et al. 2012).

In this paper, we focus on the early Swift data of this burst, with the aim of understanding its physical origin. We present our data analysis of the BAT and XRT data in Section 2, and compare
GRB 120422A with other SN-associated GRBs in Section 3. In Section 4, we then discuss the possible physical origins of prompt emission and early afterglow and constrain the bulk Lorentz factor. The results are summarized in Section 5, along with a discussion on the physical implications of this event.

\section{DATA ANALYSIS}

We processed the Swift/BAT data using standard HEAsoft tools (version 6.11). As shown in Figure 1, the main burst lasted from $T_{0}-3 \mathrm{~s}$ to $T_{0}+20 \mathrm{~s}$ with $T_{90}=5.4 \pm 1.4 \mathrm{~s}$. We extracted the BAT spectra in five time slices. The lower panel in Figure 1 shows the photon indices obtained by fitting the spectra with a simple power-law model. It is obvious that this burst has a strong hard-to-soft spectral evolution, which is similar to most other Swift/BAT GRBs. The photon indices range from $\sim 1.0$ to $\sim 2.6$. The time-integrated spectrum from 0 to $10 \mathrm{~s}$ can be fitted with a simple power law with a photon index of $\Gamma_{\mathrm{ph}}=1.94 \pm 0.3$. Weak emission (at $3 \sigma$ level) was observed at $40-65 \mathrm{~s}$ with a lowsignificance peak at $t \sim 45 \mathrm{~s}$ and a photon index of $\sim 2.1 \pm 0.7$. No significant pre-trigger emission was detected in the BAT band up to $T_{0}-200 \mathrm{~s}$.

The BAT band $(15-150 \mathrm{keV})$ peak flux is $0.6 \pm$ 0.2 photons $\mathrm{cm}^{-2} \mathrm{~s}^{-1}$, and the total fluence is about $2.3 \pm 0.4 \times$ $10^{-7} \mathrm{erg} \mathrm{cm}^{-2}$. For a burst at redshift $z=0.283$, this corresponds to a peak luminosity of $L \sim 10^{49} \mathrm{erg} \mathrm{s}^{-1}$ and a total isotropic energy of $\sim 4.5 \times 10^{49} \mathrm{erg}$. The peak luminosity is well below the typical luminosity of $\sim 10^{52} \mathrm{erg} \mathrm{s}^{-1}$ for bright GRBs, but is considerably higher than those of some nearby low-luminosity GRBs (e.g., $L \lesssim$ a few $\times 10^{47} \mathrm{erg} \mathrm{s}^{-1}$ ). 


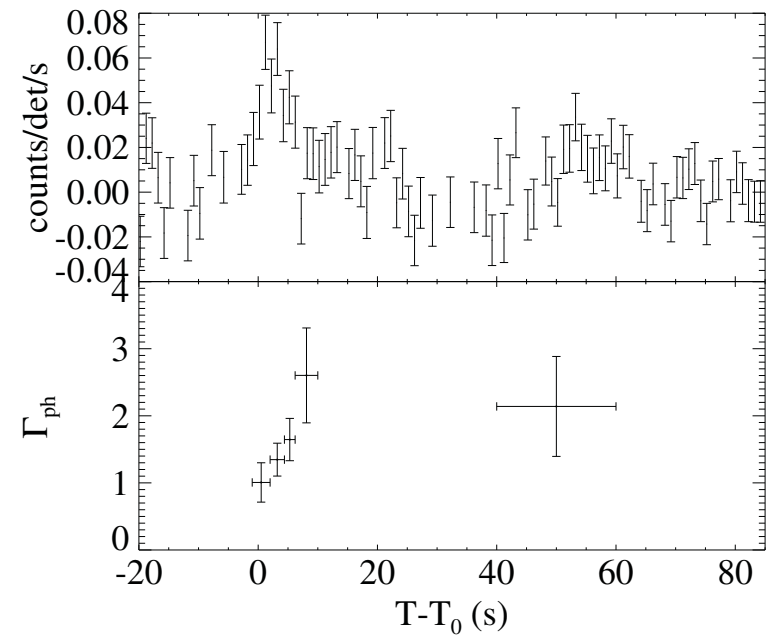

Figure 1. BAT count rate (upper panel) and photon index evolution (lower panel) of GRB 120422A. The spectral model is a simple power law ("powerlaw" in Xspec).

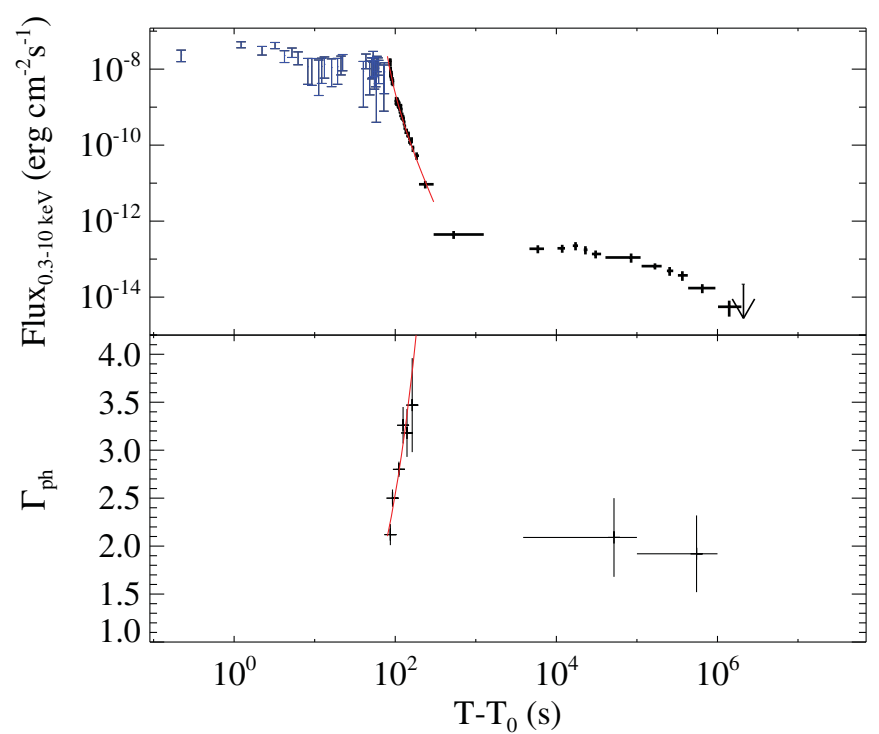

Figure 2. Upper panel: the Swift/XRT light curve of GRB 120422A (black) and the BAT light curve extrapolated to the XRT band (blue). The solid red lines show the curvature effect model (Zhang et al. 2009b) fitted to the observed flux. Lower panel: photon index evolution. The solid red line shows the curvature effect model (Zhang et al. 2009b) fitting to the observed photon index. See Section 4 for details.

(A color version of this figure is available in the online journal.)

In a standard fashion, we processed the Swift/XRT data using our own IDL code, which employs the standard HEAsoft analysis tools. For technical details please refer to Zhang et al. (2007b). Figure 2 shows the XRT light curve and spectral evolution. The XRT light curve shows an unusually steep (decay slope $>6) \mathrm{X}$-ray tail between $T_{0}+85 \mathrm{~s}$ to $T_{0}+1000 \mathrm{~s}$, followed by a shallow decay phase with a decay slope of $\sim 0.25$. A break is observed at $\sim 10^{5} \mathrm{~s}$ before the final normal decay phase (a decay slope of $\sim 1$ ). The X-ray spectrum can be fitted with an absorbed power law. Strong spectral evolution was observed in the steep decay phase where the photon indices vary significantly from $\Gamma_{\mathrm{ph}} \sim 2.1$ to $\Gamma_{\mathrm{ph}} \sim 3.5$. The latetime spectrum has no significant evolution with an average photon index of $\Gamma_{\mathrm{ph}} \sim 2.1$. The total fluence in the XRT band $\left(0.3-10 \mathrm{keV}\right.$ ) is $1.53 \pm 0.26 \times 10^{-7} \mathrm{erg} \mathrm{cm}^{-2}$ (from $\sim 86.3 \mathrm{~s}$ to $10^{6} \mathrm{~s}$, corrected for XRT observation gaps).
Table 1

The Observational Properties of GRB 120422A and Other Supernova GRBs

\begin{tabular}{|c|c|c|c|c|c|}
\hline GRB & $z$ & $\begin{array}{l}T_{90} \\
\text { (s) }\end{array}$ & $\begin{array}{c}E_{\text {peak }} \\
(\mathrm{keV})\end{array}$ & $\begin{array}{c}E_{\gamma, \text { iso }} \\
\left(10^{51} \mathrm{erg}\right)\end{array}$ & Ref. $^{\text {a }}$ \\
\hline \multicolumn{6}{|c|}{ Gold $^{\mathrm{b}}$} \\
\hline 980425 & 0.0085 & $34.9 \pm 3.8$ & $122 \pm 17$ & $9 \times 10^{-4}$ & 1,2 \\
\hline 030329 & 0.1685 & 22.9 & $70 \pm 2$ & 13 & 1 \\
\hline 031203 & 0.1055 & $37.0 \pm 1.3$ & $>190$ & 0.17 & 1,3 \\
\hline 060218 & 0.0334 & $2100 \pm 100$ & $4.7 \pm 1.2$ & 0.04 & 1,2 \\
\hline $100316 \mathrm{D}$ & 0.0591 & $>1300$ & $18_{-2}^{+3}$ & 0.06 & 1,4 \\
\hline $120422 \mathrm{~A}$ & 0.283 & $5.35 \pm 1.4$ & $\sim 53^{\mathrm{c}}$ & 0.045 & 5,6 \\
\hline \multicolumn{6}{|c|}{ Silver $^{\mathrm{b}}$} \\
\hline 011121 & 0.362 & $\sim 28$ & & 27 & 7 \\
\hline 020903 & 0.251 & $\sim 20$ & $\sim 2$ & 0.011 & 8,9 \\
\hline 021211 & 1.006 & $\sim 4$ & $46.8_{-5.1}^{+5.8}$ & 6.6 & 10 \\
\hline $050525 \mathrm{~A}$ & 0.606 & $8.8 \pm 0.5$ & $84.1 \pm 1.7$ & 23 & 11 \\
\hline 081007 & 0.5295 & 8 & $61 \pm 15$ & 1.5 & 12 \\
\hline 101219B & 0.55 & 51 & $70 \pm 8$ & 4.2 & 13 \\
\hline
\end{tabular}

Notes.

a References. (1) Hjorth \& Bloom 2011; (2) Zhang 2008; (3) Sazonov et al. 2004; (4) Sakamoto et al. 2010; (5) Barthelmy et al. 2012; (6) Schulze et al. 2012; (7) Garnavich et al. 2003; (8) Sakamoto et al. 2004; (9) Soderberg et al. 2004; (10) Crew et al. 2003; (11) Blustin et al. 2006; (12) Z. P. Jin et al. 2012, in preparation; (13) Sparre et al. 2011.

b The Gold sample includes Type II GRBs that have a spectroscopically identified supernova association and well-monitored supernova emission. The Silver sample includes GRBs that have a clear supernova bump along with some spectroscopic evidence. The similar categorization was also adopted by Hjorth \& Bloom (2011).

${ }^{\mathrm{c}}$ Estimated using the $E_{p}-\Gamma_{\mathrm{ph}}$ relation in Zhang et al. (2007a).

\section{COMPARISON WITH OTHER SUPERNOVA GRBS}

As shown in Table 1, among the bursts associated with a well-monitored SN, GRB 120422A distinguishes itself by the following facts: (1) it has the shortest $T_{90}$, (2) the initial luminosity of the X-ray radiation is high (e.g., greater than that of GRB 060218 and GRB 100316D by a factor of 100, see Figure 3) and the temporal decay slope is steep, and (3) the X-ray afterglow plateau is also significantly brighter than GRB 060218 and GRB $100316 \mathrm{D}$ in the same time frame (i.e., $10^{4}-10^{5} \mathrm{~s}$ ), even though the total prompt emission $\gamma / \mathrm{X}$-ray energies of these three bursts are comparable. This suggests that a much higher energy is carried by the relativistic outflow in GRB 120422A. In fact, among the bursts with a well-monitored spectroscopic SN detected so far (the "Gold" sample in Table 1), at one day after the burst, the X-ray afterglow of GRB 120422A is only dimmer than that of GRB 030329, a typical high-luminosity GRB in a nearby universe. (4) There is a large offset ( $\sim 8 \mathrm{kpc}$; Tanvir et al. 2012) between the burst location and the center of its host galaxy, which is rather unusual for massive star core-collapse GRBs (see, e.g., Fruchter et al. 2006; Zhang et al. 2009a). Within the Gold sample of SN GRBs, the isotropic gamma-ray energy $E_{\gamma, \text { iso }}$ of GRB 031203, GRB 060218, GRB 100316D, and GRB $120422 \mathrm{~A}$ is rather similar. Interestingly, they seem to belong to two sub-classes. As has already been noted elsewhere (e.g., Fan et al. 2011; Starling et al. 2011), XRF 060218 and XRF $100316 \mathrm{D}$ are cousins, since both their spectral and temporal behaviors are rather similar (see also Figure 3), except that the former was associated with a less energetic SN 2006aj. On the other hand, GRB 120422A and GRB 031203 (Mazzali et al. 2006) share quite a few similarities. For example, they are both relatively short, their peak luminosities during the 


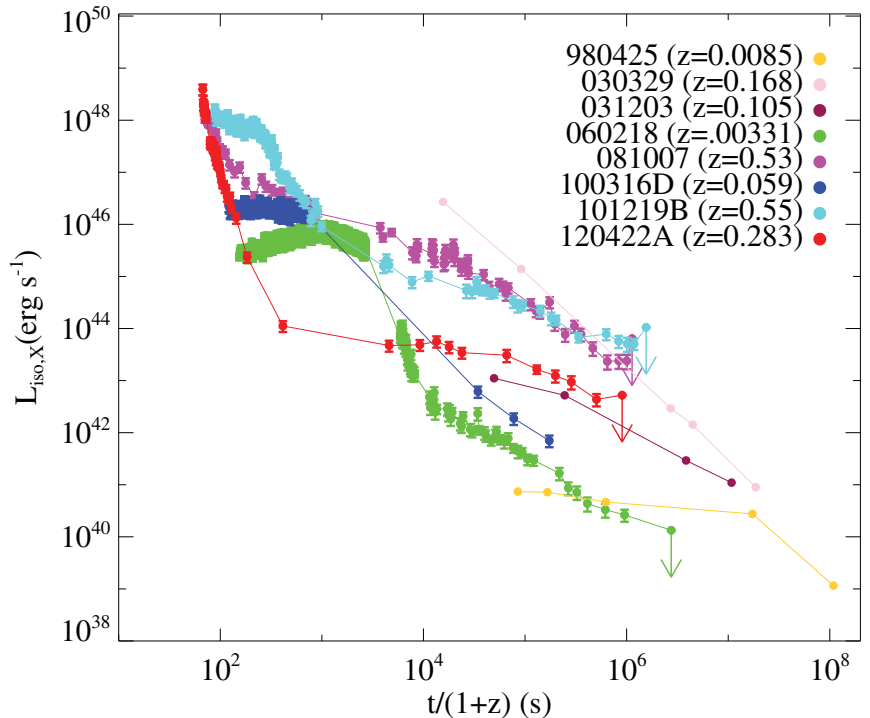

Figure 3. Comparison of the observed X-ray luminosity light curves of GRB 120422A and other supernova GRBs. The data of GRB 980425, GRB 030329, GRB 031203, GRB 060218, and XRF 100316D are the same as those of Figure 2 of Fan et al. (2011). The data from GRB 081007, GRB 101219B, and GRB $120422 \mathrm{~A}$ are analyzed in this work.

(A color version of this figure is available in the online journal.)

prompt emission phase are almost identical, their $15-150 \mathrm{keV}$ spectra are both soft with spectral indices of $\alpha \sim 0.6-0.9$ ( $\alpha$ is defined as $\left.f_{v} \propto v^{-\alpha}\right)$, and their late ( $t>1$ day) X-ray afterglow luminosities are comparable to each other, but are significantly brighter than GRB 060218 and XRF 100316D.

\section{X-RAY AFTERGLOW MODELING AND ENGINE-DRIVEN GRB}

\subsection{The Steep Decay Phase}

The steep decay phase is commonly observed in Swift GRBs (e.g., Tagliaferri et al. 2005; Barthelmy et al. 2005). The standard interpretation of this phase is the "curvature" tail of the prompt emission (Fenimore et al. 1996; Kumar \& Panaitescu 2000; Zhang et al. 2006, 2009b; Liang et al. 2006), which arises from delayed photon emission from high latitudes with respect to the line of sight upon the abrupt cessation of the prompt emission. Other interpretations include a rapid expansion of thermal plasma associated with a shock breakout or a hot cocoon surrounding a jet after exiting the progenitor star (e.g., Fan et al. 2006; Pe'er et al. 2006).

In the shock-breakout picture (Fan et al. 2006), a quick decline in X-rays is possible for a quasi-thermal spectrum $F_{v_{\text {obs }}} \propto R^{2} e^{-h v_{\text {obs }} / k T_{\text {obs }}}$ for $h v_{\text {obs }} \gg k T_{\text {obs. }}$. The temperature drops with time as $T_{\mathrm{obs}} \propto R^{-a / 3}$, where $a=2$ if the width of the hot material is fixed, or $a=3$ if the width of the hot material is proportional to the radius $R$. Taking $a=3$ as an example, i.e., $T_{\text {obs }} \propto R^{-1} \propto t^{-1}$, the XRT-band luminosity can be expressed as

$$
L_{\mathrm{XRT}} \propto \int_{0.3 \mathrm{keV}}^{10 \mathrm{keV}} F_{v_{\mathrm{obs}}} d \nu_{\mathrm{obs}} \propto F(t) e^{-A t},
$$

where $A>0$ is a constant and $F(t)>0$ is a function of $t$. In principle, this model can give rise to a progressively steep decay phase with rapid spectral evolution (for $k T<0.3 \mathrm{keV}$ ). This model does not fit the data. Also the emergence of a shallow decay component is not expected within such a scenario.

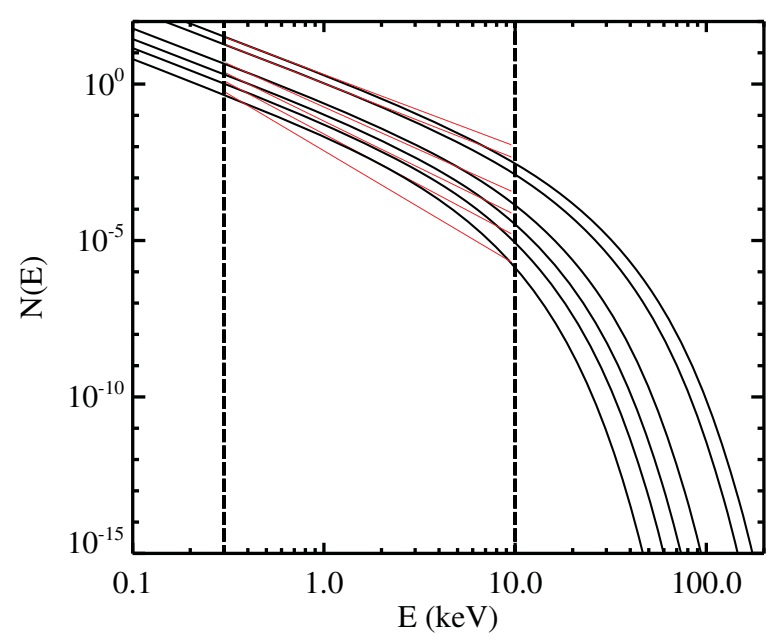

Figure 4. Time-dependent theoretical spectra based on the curvature effect of a non-power-law spectrum. From top to bottom, each spectrum corresponds to a time slice of the steep decay phase, which is the same as that in the lower panel of Figure 2. The XRT band $(0.3-10 \mathrm{keV})$ is bracketed by two vertical lines. The red solid lines show the effective power-law model in the narrow XRT band.

(A color version of this figure is available in the online journal.)

We then investigate the curvature effect model for a nonpower-law spectrum (Zhang et al. 2009b). We consider a timedependent cutoff power-law photon spectrum taking the form

$$
N(E, t)=N_{0}(t)\left(\frac{E}{1 \mathrm{keV}}\right)^{-\Gamma_{\mathrm{ph}}} e^{-\frac{E}{E_{c}(t)}},
$$

where $\Gamma_{\mathrm{ph}}$ is the power-law photon spectral index, $E_{c}(t)=$ $E_{c, p}\left[\left(t-t_{0}\right) /\left(t_{p}-t_{0}\right)\right]^{-1}$ is the time-dependent characteristic cutoff photon energy, $N_{0}(t)=N_{0, p}\left[\left(t-t_{0}\right) /\left(t_{p}-t_{0}\right)\right]^{-\left(1+\Gamma_{\mathrm{ph}}\right)}$ is a time-dependent photon flux, and $t_{0}$ refers to the time origin of the last/main pulse in the prompt emission. Denoting $t_{p}$ as the peak time of the last pulse, one can derive the time-dependent decay index and the effective spectral index using the formalism derived in Zhang et al. (2009b). For GRB 120422A, $t_{p}$ cannot be inferred from the XRT light curve, since the X-ray had already entered the steep decay phase when the XRT slewed to the source. To constrain $t_{p}$, we extrapolate the BAT flux to the XRT band assuming a simple power-law model extending all the way to the XRT band. It is found that the BAT flux extrapolated to the XRT band and the observed XRT light curve intersect around the time when the XRT observation started (Figure 2). We thus take $t_{p} \sim 86.3 \mathrm{~s}$ (the beginning of XRT observation) in our modeling.

We successfully fit both the observed light curve and the photon index curve with our model, and get the following bestfit parameters: $N_{0, p}=2.36 \pm 0.09, E_{c, p}=7.62_{-0.83}^{+0.97} \mathrm{keV}$, $\Gamma_{\mathrm{ph}}=2.30 \pm 0.07, t_{0}=57.5 \pm 0.65 \mathrm{~s}$, with $\chi^{2} /$ dof $=71.2 / 52$ (Figure 2). Figure 4 gives the modeled spectra as a function of time. This suggests that there was likely a central-enginepowered emission in the time interval $58 \mathrm{~s}-86 \mathrm{~s}$. Together with the main activity in the first $\sim 20 \mathrm{~s}$ and the weak/soft radiation from $40 \mathrm{~s}$ to $60 \mathrm{~s}$, the variability of the prompt emission of GRB 120422A is well established. This strongly favors a central engine origin of the observed prompt emission.

For high-latitude emission, a rough constraint on the emission radius, and hence, bulk Lorentz factor $\Gamma$ of the outflow (within the framework of the internal shock model) may be imposed (e.g., Zhang et al. 2006; Jin et al. 2010). The length of the tail emission $t_{\text {tail }}=t-t_{p}$ can be expressed as 
$t_{\text {tail }} \leqslant 2 \Gamma^{2} \Delta t\left(1-\cos \theta_{j}\right)$, where $\Delta t \sim t_{p}-t_{0}$ is the variability timescale and $\theta_{j}$ is the jet opening angle. Plugging in the numbers, i.e., $t_{\text {tail }} \sim 250-86=164 \mathrm{~s}, \Delta t=29 \mathrm{~s}$, one can derive a constraint

$$
\Gamma \geqslant \frac{1.68}{\sqrt{1-\cos \theta_{j}}} .
$$

For $\theta_{j}=10^{\circ}, 20^{\circ}, 30^{\circ}$, the corresponding constraints are $\Gamma \geqslant 13.6,6.8,4.6$, respectively.

\subsection{The Plateau Phase}

Following the steep decay phase is an X-ray plateau, lasting until $\sim 1$ day after the trigger. This component is commonly observed in high-luminosity GRBs, and there is no consensus regarding its interpretation. We discuss the following two possible interpretations.

Scenario I. The X-ray plateau is due to the forward shock emission of a mildly relativistic outflow during the "coasting phase" before significant deceleration starts (e.g., Shen \& Matzner 2012). For a wind medium with density profile $n=$ $3 \times 10^{35} \mathrm{~cm}^{-2} A_{*} r^{-2}$, one can show that the decay rate is very shallow in this phase, i.e., $F_{v} \propto t^{-(p-2) / 2} \propto t^{-(\beta-1)}$, if the $\mathrm{X}$-ray band frequency satisfies $v>\max \left(v_{m}, v_{c}\right)$. The postdeceleration decay behavior in the same spectrum regime is $F_{v} \propto t^{-(3 p-2) / 4} \propto t^{-(3 \beta-1) / 2}$. Both behaviors are in agreement with the data.

This interpretation leads to the following constraints: (1) the outflow deceleration time $t_{\mathrm{dec}}=t_{b}$, where $t_{b}=10^{5} \mathrm{~s}$, is the shallow-to-normal break time, (2) the external forward shock flux density at $t_{b}$ is measured as $F_{v_{x}}\left(t_{b}\right)=1.25 \times 10^{-2} \mu \mathrm{Jy}$, (3) $v_{m}\left(t_{1}\right) \leqslant v_{x}$, and (4) $v_{c}\left(t_{2}\right) \leqslant v_{x}$, where $t_{1}=10^{3} \mathrm{~s}$ and $t_{2}=10^{6} \mathrm{~s}$ are the observed starting time of the shallow decay and the lower limit of the end time of normal decay, respectively. The last two constraints are set in order to satisfy the spectral regime requirement $v>\max \left(v_{m}, v_{c}\right)$ for both the plateau and the normal decay phase, and utilize the model prediction that $v_{m}(t)$ decreases and $v_{c}(t)$ increases with $t$ monotonically. We follow the formulae in Shen \& Matzner (2012, Equations (14)-(17) therein), which are based on the standard external shock synchrotron emission calculation (e.g., Sari et al. 1998) and include a numerical correction factor due to the internal structure of the shock and the equal-arrival-time surface (Granot et al. 1999). We adopt $\nu_{x}=1 \mathrm{keV}$ and use $\beta=2.1$ as observed.

Constraint (2) gives the wind medium density normalization:

$$
A_{*}=0.4 \epsilon_{e}^{-1.14} \epsilon_{B}^{-0.05} \Gamma^{-4},
$$

where $\Gamma$ is the initial Lorentz factor of the outflow, and $\epsilon_{e}$ and $\epsilon_{B}$ are the shock electron and magnetic equipartition parameters, respectively. Combining constraints (1) and (2) gives the isotropic equivalent kinetic energy of the outflow as

$$
E_{\mathrm{k}, \text { iso }}=1.2 \times 10^{51}\left(\frac{\epsilon_{e}}{0.01}\right)^{-1.14}\left(\frac{\epsilon_{B}}{0.01}\right)^{-0.05} \mathrm{erg} .
$$

Constraint (3) is trivial and easily satisfied. Utilizing Equation (4), constraint (4) gives

$$
\Gamma \leqslant 6.5\left(\frac{\epsilon_{e}}{0.01}\right)^{-0.21}\left(\frac{\epsilon_{B}}{0.01}\right)^{0.18} .
$$

This constraint is consistent with the curvature effect constraints if $\theta_{j}>20^{\circ}$. So all of the afterglow data are consistent with a wide jet with a moderately high Lorentz factor of $\Gamma \sim 6$.
Scenario II. If the jet is narrower, say $\theta_{j}<20^{\circ}$, the X-ray plateau cannot be interpreted as the pre-deceleration forward shock in a wind medium. The deceleration time has to be much earlier, and the extended plateau can be interpreted as forward shock emission with significant energy injection ${ }^{10}$ (e.g., Zhang et al. 2006, and the references therein). There are two possible cases. Case (A): one can argue that the central engine is a millisecond magnetar with a dipole radiation luminosity of $\sim 10^{47} \mathrm{erg} \mathrm{s}^{-1}$ and a spin-down timescale of $\tau_{0} \sim 10^{5} \mathrm{~s}$. This gives a constraint on the surface magnetic field of $B_{p}=$ $(0.5-1) \times 10^{14} \mathrm{G}$ and the initial spin period as $P_{0} \sim 1 \mathrm{~ms}$. One potential challenge of this scenario is that the efficiency of the forward shock radiation in the XRT band has to be extremely small (say, very low $\epsilon_{e}$ ). Otherwise, the resulting $\mathrm{X}$-ray emission would be much brighter than what is observed. Case (B): one may argue that the outflow has a Lorentz factor distribution and the distribution satisfies $E(>\Gamma) \propto \Gamma^{-5}$.

In both scenarios, the X-ray flux at $t \sim 10^{5} \mathrm{~s}$ constrains the total kinetic energy of the outflow, which is given by Equation (5). However, the total kinetic energy of the initial outflow that produces the prompt burst, $E_{\mathrm{k}, \mathrm{p} \text {,iso }}$, is different for the two scenarios. In scenario I, $E_{\mathrm{k}, \mathrm{p} \text {, iso }}=E_{\mathrm{k} \text {,iso }}$, while in scenario II, $E_{\mathrm{k}, \mathrm{p} \text {,iso }} \ll E_{\mathrm{k} \text {,iso }}$.

\section{CONCLUSIONS AND DISCUSSION}

We have analyzed the BAT and XRT data of the nearby, lowluminosity, SN-associated GRB 120422A. Even though $T_{90}$ of the burst is short, BAT emission shows extended fluctuation signals, suggesting a possible extended central engine activity. This is confirmed by the XRT data, which showed a rapid decline followed by an extended plateau similar to most other high-luminosity GRBs. The rapid decline tail can be modeled by the curvature effect model of Zhang et al. (2009b). The derived beginning time of the last emission episode is about $58 \mathrm{~s}$, with the last peak near $86 \mathrm{~s}$. Various arguments (see below for more discussion) suggest that this low-luminosity GRB is central-engine-driven, rather than powered by a shock breakout. The Lorentz factor of the ejecta is constrained to be at least moderately relativistic.

As discussed above, an engine-driven origin is supported by the following facts: (1) the $\gamma$-ray light curve is variable, (2) the rapidly decaying prompt tail emission is inconsistent with a cooling thermal emission component from a shock breakout, but is consistent with the curvature tail of a successful jet, and (3) a long lasting X-ray shallow decay followed by a steep decay is consistent with external shock emission of a successful jet.

Some nearby low-luminosity GRBs may have the signature of a shock breakout (e.g., GRB 060218; Campana et al. 2006; Waxman et al. 2007, but see Ghisellini et al. 2006, 2007a; Li 2007; Björnsson 2008; Chevalier \& Fransson 2008; Page et al. 2011). The event rate of nearby low-luminosity GRBs is much higher than the simple extrapolation of the high-luminosity GRB event rate, making a distinct population (e.g., Soderberg et al. 2006; Liang et al. 2007; Virgili et al. 2009; Coward 2005). Some authors have suspected that low-luminosity GRBs may be unsuccessful jets, and the radiation signal is mostly powered by a shock breakout. The relativistic shock-breakout model predicts a "fundamental plane" correlation of $T_{90} \sim$ $20 \mathrm{~s}(1+z)^{-1.68}\left(E_{\gamma, \text { iso }} / 10^{46} \mathrm{erg}\right)^{1 / 2}\left(E_{\mathrm{p}} / 50 \mathrm{keV}\right)^{-2.68}($ Nakar \& Sari 2012). For the parameters of this burst, $E_{\gamma \text {,iso }} \sim 4 \times 10^{49} \mathrm{erg}$

\footnotetext{
10 An alternative solution is to explain the plateau phase as late prompt
} emission (see, e.g., Ghisellini et al. 2007b). 


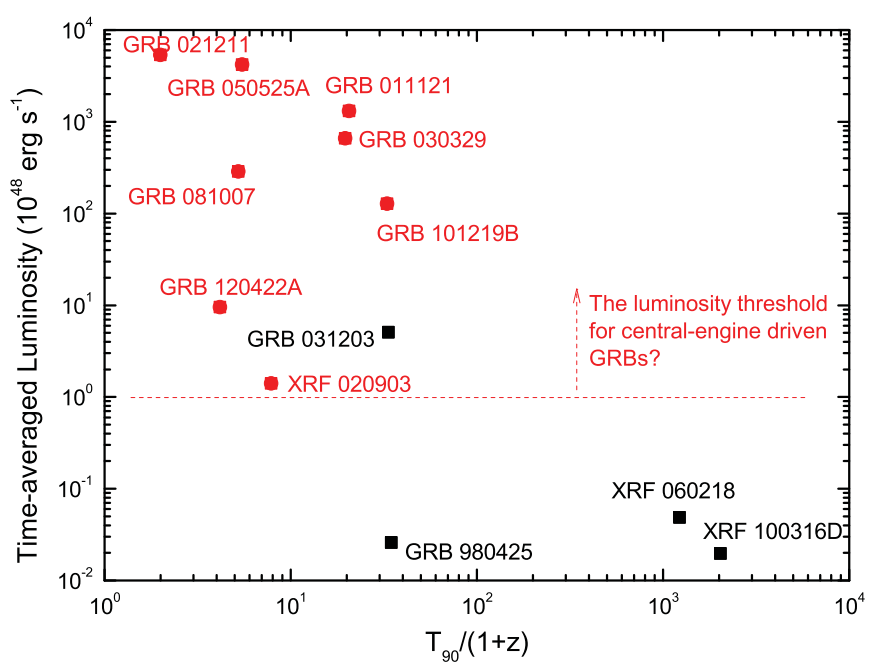

Figure 5. Supernova-associating GRBs in the time-averaged luminosity- $T_{90} /(1+z)$ plane. The red symbols denote engine-driven GRBs, while the black ones denote the possible shock-breakout GRBs suggested in some literature. The red dashed line $\left(10^{48} \mathrm{erg} \mathrm{s}^{-1}\right)$ gives a rough threshold above which successful a jet is possible.

(A color version of this figure is available in the online journal.)

and $E_{\mathrm{p}} \sim 53 \mathrm{keV}$, the predicted shock-breakout duration is of $\sim 1100 \mathrm{~s}$, much longer than $T_{90} \sim 5 \mathrm{~s}$, or the extended duration of $\sim 86$ derived from the curvature effect fitting. This is strong evidence against the shock-breakout interpretation of this burst.

In the collapsar model for GRBs, in order to make a successful jet, the central engine has to be active for a duration longer than the time required for the jet to penetrate the star before breaking out. Otherwise the jet would be choked inside the star or quickly spread out upon the breakout. Considering the collimation of the jet by a surrounding cocoon, Bromberg et al. (2011) estimate the breakout time as

$$
\begin{aligned}
t_{\mathrm{B}} \simeq & 15 \epsilon_{\gamma}^{1 / 3}\left(\frac{L_{\gamma, \text { iso }}}{10^{50} \mathrm{erg} \mathrm{s}^{-1}}\right)^{-1 / 3}\left(\frac{\theta_{0}}{10^{\circ}}\right)^{2 / 3} \\
& \times\left(\frac{R_{*}}{10^{11} \mathrm{~cm}}\right)^{2 / 3}\left(\frac{M_{*}}{15 M_{\odot}}\right)^{1 / 3} s,
\end{aligned}
$$

where $\epsilon_{\gamma}$ is the burst radiation efficiency, and $\theta_{0}$ is the initial opening angle of the jet when it is injected from the central engine. Statistically, one would expect the observed burst duration to be comparable to or longer than this duration. For GRB 120422A, even if $T_{90} \sim 5$ is shorter than this jet penetration time, the real duration of the successful jet is actually near $86 \mathrm{~s}$, as it is constrained by the curvature effect modeling. The jet breakout condition is therefore satisfied.

What is the separation line between the engine-driven and the shock-break GRBs? In Figure 5 all the SN GRBs are plotted in the plane of time-averaged luminosity and $T_{90}$. It is shown that above $\sim 10^{48} \mathrm{erg} \mathrm{s}^{-1}$, an engine-driven GRB is possible. Shockbreakout luminosity cannot be much higher than this value. Therefore GRB 120422A belongs to the low end of enginedriven GRBs.

How could a successful GRB jet have such a low luminosity? The first possibility may be related to its relatively low Lorentz factor (scenario I of the plateau interpretation). If this burst satisfies the empirical $\Gamma-E_{\gamma}$,iso and $\Gamma-L_{\gamma}$,iso relations (Liang et al. 2010; Lü et al. 2012; Fan et al. 2012), one would expect a moderately low $\Gamma$. This is generally consistent with the model constraints of $\Gamma$. Low- $\Gamma$ outflows tend to have low emissivities.
This can be due to an intrinsically low wind luminosity, or a smaller radiation efficiency for an otherwise normal wind luminosity. This second possibility can be related to the internal shock model when the relative Lorentz factor between the colliding shells is small (e.g., Barraud et al. 2005). Alternatively, the low luminosity can be related to the viewing angle effect. A low-luminosity GRB can be obtained by an observer viewing the jet axis of a structured jet at a large angle (e.g., Zhang et al. 2004a). This may be relevant for a hot cocoon surrounding a successful jet (e.g., Zhang et al. 2004b), which is consistent with the low- $\Gamma$, large $\theta_{j}$ scenario discussed in this paper. This scenario can be tested with the late-time radio observations, which would give a more robust measure of the total energetics of the event.

We thank Derek B. Fox, Péter Mészáros, Dirk Grupe, and Péter Veres for helpful discussions. This work is supported in part by NASA SAO SV4-74018 (B.B.Z.), the National Natural Science Foundation of China (grants 10973041, 10921063, 11073057, and 11163003) and the National Basic Research Program of China under grant 2009CB824800, the 100 Talents Program of Chinese Academy of Sciences (Y.Z.F.), NASA NNX10AD08G, and NSF AST-0908362 (B.Z.).

\section{REFERENCES}

Barraud, C., Daigne, F., Mochkovitch, R., \& Atteia, J. L. 2005, A\&A, 440, 809 Barthelmy, S. D., Barbier, L. M., Cummings, J. R., et al. 2005, Space Sci. Rev., 120,143

Barthelmy, S. D., Baumgartner, W. H., Cummings, J. R., et al. 2012, GCN Circ., 13246

Beardmore, A. P., Evans, P. A., Goad, M. R., \& Osborne, J. P. 2012, GCN Circ., 13247

Björnsson, C.-I. 2008, ApJ, 672, 443

Blustin, A. J., Band, D., Barthelmy, S., et al. 2006, ApJ, 637, 901

Bromberg, O., Nakar, E., Piran, T., \& Sari, R. 2011, ApJ, 740, 100

Burrows, D. N., Hill, J. E., Nousek, J. A., et al. 2005, Space Sci. Rev., 120, 165

Campana, S., Mangano, V., Blustin, A. J., et al. 2006, Nature, 442, 1008

Chevalier, R. A., \& Fransson, C. 2008, ApJ, 683, L135

Coward, D. M. 2005, MNRAS, 360, L77

Crew, G. B., Lamb, D. Q., Ricker, G. R., et al. 2003, ApJ, 599, 387

Fan, Y. Z., Piran, T., \& Xu, D. 2006, J. Cosmol. Astropart. Phys., JCAP09(2006)013

Fan, Y.-Z., Wei, D.-M., Zhang, F.-W., \& Zhang, B.-B. 2012, ApJ, 755, L6 Fan, Y. Z., Zhang, B. B., Xu, D., Liang, E. W., \& Zhang, B. 2011, ApJ, 726, 32 Fenimore, E. E., Madras, C. D., \& Nayakshin, S. 1996, ApJ, 473, 998 Fruchter, A. S., Levan, A. J., Strolger, L., et al. 2006, Nature, 441, 463 Garnavich, P. M., Stanek, K. Z., Wyrzykowski, L., et al. 2003, ApJ, 582, 924 Ghisellini, G., Ghirlanda, G., Mereghetti, S., et al. 2006, MNRAS, 372, 1699 Ghisellini, G., Ghirlanda, G., Nava, L., \& Firmani, C. 2007a, ApJ, 658, L75 Ghisellini, G., Ghirlanda, G., \& Tavecchio, F. 2007b, MNRAS, 375, L36 Granot, J., Piran, T., \& Sari, R. 1999, ApJ, 513, 679

Hjorth, J., \& Bloom, J. S. 2011, in Gamma-ray Bursts, ed. C. Kouveliotou, R. A. M. J. Wijers, \& S. E. Woosley (Cambridge: Cambridge Univ. Press), chapter 9

Jin, Z. P., Fan, Y. Z., \& Wei, D. M. 2010, ApJ, 724, 861

Kuin, N. P. M., \& Troja, E. 2012, GCN Circ., 13248

Kumar, P., \& Panaitescu, A. 2000, ApJ, 541, L51

Liang, E., Zhang, B., Virgili, F., \& Dai, Z. G. 2007, ApJ, 662, 1111

Liang, E.-W., Yi, S.-X., Zhang, J., et al. 2010, ApJ, 725, 2209

Liang, E.-W., Zhang, B., O’Brien, P. T., et al. 2006, ApJ, 646, 351

Li, L.-X. 2007, MNRAS, 375, 240

Lü, J., Zou, Y.-C., Lei, W.-H., et al. 2012, ApJ, 751, 49

Malesani, D., Schulze, S., Kruehler, T., et al. 2012a, GCN Circ., 13275

Malesani, D., Schulze, S., Kruehler, T., et al. 2012b, GCN Circ., 13277

Mazzali, P. A., Deng, J., Pian, E., et al. 2006, ApJ, 645, 1323

Melandri, A., Pian, E., Ferrero, P., et al. 2012, arXiv:1206.5532

Nakar, E., \& Sari, R. 2012, ApJ, 747, 88

Nardini, M., Schmidl, S., Greiner, J., \& Kann, D. A. 2012, GCN Circ., 13256

Page, K. L., Starling, R. L. C., Fitzpatrick, G., et al. 2011, MNRAS, 416, 2078

Pe'er, A., Mészáros, P., \& Rees, M. J. 2006, ApJ, 652, 482 
Roming, P. W., Kennedy, T. E., Mason, K. O., et al. 2005, Space Sci. Rev., 120, 95

Rumyantsev, V., Antonyuk, K., \& Pozanenko, A. 2012, GCN Circ., 13273

Sakamoto, T., Barthelmy, S. D., Baumgartner, W. H., et al. 2010, GCN Circ., 10511

Sakamoto, T., Lamb, D. Q., Graziani, C., et al. 2004, ApJ, 602, 875

Sanchez-Ramirez, R., Leloudas, G., de Ugarte Postigo, A., et al. 2012, GCN Circ., 13281

Sari, R., Piran, T., \& Narayan, R. 1998, ApJ, 497, L17

Sazonov, S. Y., Lutovinov, A. A., \& Sunyaev, R. A. 2004, Nature, 430, 646

Schulze, S., Levan, A. J., Malesani, D., et al. 2012, GCN Circ., 13257

Shen, R. F., \& Matzner, C. D. 2012, ApJ, 744, 36

Soderberg, A. M., Kulkarni, S. R., Berger, E., et al. 2004, ApJ, 606, 994

Soderberg, A. M., Kulkarni, S. R., Nakar, E., et al. 2006, Nature, 442, 1014

Sparre, M., Sollerman, J., Fynbo, J. P. U., et al. 2011, ApJ, 735, L24

Starling, R. L. C., Wiersema, K., Levan, A. J., et al. 2011, MNRAS, 411, 2792
Tagliaferri, G., Goad, M., Chincarini, G., et al. 2005, Nature, 436, 985

Tanvir, N. R., Levan, A. J., Cucchiara, A., \& Fox, D. B. 2012, GCN Circ., 13251

Troja, E., D’Elia, V., Guidorzi, C., et al. 2012, GCN Circ., 13243

Virgili, F., Liang, E.-W., \& Zhang, B. 2009, MNRAS, 392, 91

Waxman, E., Mészáros, P., \& Campana, S. 2007, ApJ, 667, 351

Wiersema, K., Cucchiara, A., Levan, A. J., et al. 2012, GCN Circ., 13276

Zhang, B., Dai, X., Lloyd-Ronning, N. M., \& Mészáros, P. 2004a, ApJ, 601, L119

Zhang, B., Fan, Y. Z., Dyks, J., et al. 2006, ApJ, 642, 354

Zhang, B., Zhang, B.-B., Liang, E.-W., et al. 2007a, ApJ, 655, L25

Zhang, B., Zhang, B.-B., Virgili, F., et al. 2009a, ApJ, 703, 1696

Zhang, B. B., Liang, E. W., \& Zhang, B. 2007b, ApJ, 666, 1002

Zhang, B. B., Zhang, B., Liang, E. W., \& Wang, X. Y. 2009b, ApJ, 690, L10

Zhang, F.-W. 2008, ApJ, 685, 1052

Zhang, W., Woosley, S. E., \& Heger, A. 2004b, ApJ, 608, 365 